\title{
Is GW190521 the merger of black holes from the first stellar generations?
}

\author{
Eoin Farrell ${ }^{\circledR},{ }^{1 \star}$ Jose H. Groh, ${ }^{1}$ Raphael Hirschi, ${ }^{2,3}$ Laura Murphy, ${ }^{1}$ Etienne Kaiser ${ }^{\circledR},{ }^{2}$ Sylvia Ekström, \\ Cyril Georgy ${ }^{4}$ and Georges Meynet ${ }^{4}$ \\ ${ }^{1}$ School of Physics, Trinity College Dublin, The University of Dublin, Dublin 2, Ireland \\ ${ }^{2}$ Astrophysics Group, Keele University, Keele, Staffordshire ST5 5BG, UK \\ ${ }^{3}$ Institute for the Physics and Mathematics of the Universe (WPI), University of Tokyo, 5-1-5 Kashiwanoha, Kashiwa 277-8583, Japan \\ ${ }^{4}$ Geneva Observatory, University of Geneva, Chemin des Maillettes 51, CH-1290 Sauverny, Switzerland
}

Accepted 2020 December 1. Received 2020 November 20; in original form 2020 September 14

\begin{abstract}
GW190521 challenges our understanding of the late-stage evolution of massive stars and the effects of the pair instability in particular. We discuss the possibility that stars at low or zero metallicity could retain most of their hydrogen envelope until the pre-supernova stage, avoid the pulsational pair-instability regime, and produce a black hole with a mass in the mass gap by fallback. We present a series of new stellar evolution models at zero and low metallicity computed with the GENEVA and MESA stellar evolution codes and compare to existing grids of models. Models with a metallicity in the range 0-0.0004 have three properties that favour higher black hole (BH) masses. These are (i) lower mass-loss rates during the post main sequence phase, (ii) a more compact star disfavouring binary interaction, and (iii) possible $\mathrm{H}-\mathrm{He}$ shell interactions which lower the $\mathrm{CO}$ core mass. We conclude that it is possible that GW190521 may be the merger of black holes produced directly by massive stars from the first stellar generations. Our models indicate $\mathrm{BH}$ masses up to $70-75 \mathrm{M}_{\odot}$. Uncertainties related to convective mixing, mass loss, $\mathrm{H}-\mathrm{He}$ shell interactions, and pair-instability pulsations may increase this limit to $\sim 85 \mathrm{M}_{\odot}$.
\end{abstract}

Key words: stars: evolution-stars: massive-stars: mass-loss -stars: black holes.

\section{INTRODUCTION}

The binary black hole merger GW190521 reported by the LIGO VIRGO Collaboration (The LIGO Scientific Collaboration 2020a, b) contains unusually high component masses of $85_{-14}^{+21}$ and $66_{-18}^{+17} \mathrm{M}_{\odot}$. These black hole masses lie within the mass gap predicted by standard (pulsational) pair-instability supernova (PISN) theory. In this Letter, we investigate the possibility that stars at low or zero metallicity could retain most of their hydrogen envelope until the pre-supernova stage, avoid the pulsational pair-instability regime, and produce a black hole with a mass in the pair-instability mass gap.

In stars with $\mathrm{CO}$ core masses, $M_{\mathrm{CO}} \gtrsim 30 \mathrm{M}_{\odot}$, the late nuclear burning phases are expected to be interrupted by the production of electron-positron pairs in the core (Fowler \& Hoyle 1964; Rakavy, Shaviv \& Zinamon 1967). For stars with CO core masses of $30 \mathrm{M}_{\odot} \lesssim$ $M_{\mathrm{CO}} \lesssim 60 \mathrm{M}_{\odot}$, this can result in a series of energetic pulses followed by a collapse to a $\mathrm{BH}$ called a pulsational pair instability supernovae (PPISN) (Chatzopoulos \& Wheeler 2012; Chen et al. 2014; Woosley 2017; Marchant et al. 2019; Leung, Nomoto \& Blinnikov 2019). For $60 \mathrm{M}_{\odot} \lesssim M_{\mathrm{CO}} \lesssim 120 \mathrm{M}_{\odot}$, pair creation can result in a complete disruption of the star in a PISN, leaving behind no remnant (Glatzel, Fricke \& El Eid 1985; Fryer, Woosley \& Heger 2001; Umeda \& Nomoto 2002; Kasen, Woosley \& Heger 2011). For even higher $M_{\mathrm{CO}}$, energy losses due to photodisintegration are expected to result in a direct collapse to a BH (Fowler \& Hoyle 1964; Ober, El Eid \& Fricke 1983; Heger et al. 2003; Woosley, Blinnikov \& Heger 2007).

^E-mail: efarrel4@tcd.ie
The combined effect of PPI and PI is predicted to produce a gap in the BH birth mass distribution between $\sim 55$ and $130 \mathrm{M}_{\odot}$ (Heger et al. 2003; Belczynski et al. 2016; Woosley 2019; Giacobbo, Mapelli \& Spera 2018). The exact boundaries of the mass gap are uncertain due to uncertainties in stellar evolution, core-collapse supernovae, PPISNe, and PISNe (Woosley 2017; Farmer et al. 2019; Marchant et al. 2019; Stevenson et al. 2019; Mapelli et al. 2020; Renzo et al. 2020). Farmer et al. (2019) found that the lower boundary of the mass gap is quite robust against uncertainties in the metallicity $\left(\sim 3 \mathrm{M}_{\odot}\right)$, internal mixing $\left(\sim 1 \mathrm{M}_{\odot}\right)$, and stellar wind mass-loss $\left(\sim 4 \mathrm{M}_{\odot}\right)$. However, they found that varying the ${ }^{12} \mathrm{C}(\alpha, \gamma){ }^{16} \mathrm{O}$ reaction rate within $1 \sigma$ uncertainties shifts the location of the lower-boundary of the mass gap between 40 and $56 \mathrm{M}_{\odot}$. van Son et al. (2020) investigated the possibility of super-Eddington accretion forming $\mathrm{BHs}$ in the mass gap, however they found no binary black hole (BBH) with a combined mass $>100 \mathrm{M}_{\odot}$. Additionally, Marchant \& Moriya (2020) investigated the impact of stellar rotation on the location of the mass gap and found that the lower boundary may be shifted upwards by $4-15$ per cent depending on the efficiency of angular momentum transport. The boundaries of the pair-instability mass gap have also been proposed as a mechanism to place constraints on nuclear reaction rates (Farmer et al. 2020), particle physics (Croon, McDermott \& Sakstein 2020), and in cosmological studies (Farr et al. 2019).

Based on the $\mathrm{BH}$ mass function predicted by PPISNe and PISNe, the observation of a pre-merger $\sim 85 \mathrm{M}_{\odot} \mathrm{BH}$ as in GW190521 is unexpected. Several possibilities to create black holes with the reported mass are presented in previous works. The $\mathrm{BH}$ could form 
Table 1. Summary of our stellar evolution models. CBM refers to the free parameter regulating convective boundary mixing.

\begin{tabular}{|c|c|c|c|c|c|c|}
\hline$Z$ & $\begin{array}{c}M_{\text {zams }} \\
\left(\mathrm{M}_{\odot}\right)\end{array}$ & $\begin{array}{c}\mathrm{CBM} \\
\left(\alpha_{\mathrm{ov}} / f_{\mathrm{CBM}}\right)\end{array}$ & $\begin{array}{l}\text { Mass lost } \\
\qquad\left(\mathrm{M}_{\odot}\right)\end{array}$ & $\begin{array}{l}M_{\text {final }}^{\text {tot }} \\
\left(\mathrm{M}_{\odot}\right)\end{array}$ & $\begin{array}{l}M_{\text {final }}^{\mathrm{CO}} \\
\left(\mathrm{M}_{\odot}\right)\end{array}$ & $\begin{array}{l}R_{\max } \\
\left(\mathrm{R}_{\odot}\right)\end{array}$ \\
\hline \multicolumn{7}{|c|}{ GENEC non-rotating models ( $\alpha_{\mathrm{ov}}$ value given for $\mathrm{CBM}$ ) } \\
\hline 0 & 60 & 0.1 & 0.0 & 60.0 & 24.0 & 35 \\
\hline 0 & 85 & 0.1 & 0.0 & 85.0 & 32.4 & 142 \\
\hline 0 & 120 & 0.1 & 0.0 & 120.0 & 54.4 & 219 \\
\hline \multicolumn{7}{|c|}{ GENEC rotating models $\left(\mathrm{v}=0.4 v_{\text {crit }}\right)$} \\
\hline 0 & 60 & 0.1 & 0.3 & 59.7 & 20.9 & 56 \\
\hline 0 & 85 & 0.1 & 1.0 & 84.0 & 31.3 & 90 \\
\hline 0 & 120 & 0.1 & 3.5 & 116.5 & 56.4 & 107 \\
\hline \multicolumn{7}{|c|}{ MESA models $\left(f_{\mathrm{CBM}}\right.$ value given for $\left.\mathrm{CBM}\right)$} \\
\hline $10^{-6}$ & 85 & 0.001 & 0.30 & 84.7 & 34.4 & 794 \\
\hline 0.0003 & 85 & 0.0 & 3.0 & 82 & 28.3 & 766 \\
\hline 0.0003 & 85 & 0.001 & 3.2 & 81.7 & 32.3 & 1169 \\
\hline 0.0003 & 85 & 0.01 & 3.0 & 82 & 32.0 & 672 \\
\hline 0.0003 & 85 & 0.05 & 7.0 & 78 & 51.0 & 984 \\
\hline
\end{tabular}

as a result of hierarchical mergers in dense stellar clusters, i.e. it is the result of the prior merger of two or more other BHs (e.g. Miller \& Hamilton 2002; Fishbach, Holz \& Farr 2017; Gerosa \& Berti 2017; Rodriguez et al. 2019; Fragione, Loeb \& Rasio 2020; Gayathri et al. 2020; Romero-Shaw et al. 2020). Other possible explanations include a stellar merger between a post main-sequence star and a mainsequence binary companion (Di Carlo et al. 2019; Spera et al. 2019), a primordial origin De Luca et al. (2020), different assumptions for stellar wind mass loss Belczynski et al. (2020), Population III stars in binary systems (Kinugawa, Nakamura \& Nakano 2020a, b; Tanikawa et al. 2020), an alternative prior in the gravitational wave analysis (Fishbach \& Holz 2020), and modifications to the standard model of particle physics (Sakstein et al. 2020). The LIGO Scientific Collaboration (2020b) found alternative explanations for the source of GW190521 to be highly unlikely, including a strongly gravitationally lensed merger or a highly eccentric merger. Given the widely predicted existence of the mass gap and the apparent robustness of the boundary of the gap with respect to uncertainties in stellar evolution models, can a single star produce a $\mathrm{BH}$ remnant with a mass around $85 \mathrm{M}_{\odot}$ ?

\section{STELLAR EVOLUTION MODELS}

We present a series of new stellar evolution models computed with the GENEVA Stellar Evolution code, GENEC (Ekström et al. 2012; Murphy et al. in preperation) and with MESA (r10398; Paxton et al. 2011, 2013, 2015). We also discuss the results from existing GENEC model grids (Ekström et al. 2012; Georgy et al. 2013; Groh et al. 2019). Except where otherwise stated, the input physics for the GENEC and MESA models are similar to those described in Ekström et al. (2012) and Choi et al. (2016), respectively. In our MESA models, we use the Ledoux criterion for convection with an exponential overshooting parametrized by $f_{\mathrm{CBM}}$, while in our GENEC models, we use the Schwarzschild criterion with step-overshooting parametrized by $\alpha_{\mathrm{ov}}$. In most models, we compute the evolution until at least the end of central $\mathrm{C}$ burning. For some GENEC rotating models, the computation is stopped at the end of He burning due to convergence difficulties. We define the $\mathrm{CO}$ core mass as the region where the helium abundance $Y<0.01$ at the end of the evolution. The outputs from our models are summarized in Table 1.

Fig. 1 compares the LIGO BBH masses (Abbott et al. 2019) with the final masses and $\mathrm{CO}$ core masses of our models. The $85 \mathrm{M}_{\odot}$ models with $Z$ in the range $0-0.0004$ have final masses ranging from 76 to $85 \mathrm{M}_{\odot}$ and $\mathrm{CO}$ core masses ranging from 28 to $51 \mathrm{M}_{\odot}$. In this metallicity range, the final mass depends on assumptions about convective boundary mixing and post-MS mass-loss. Not surprisingly, the model with the lowest amount of convective boundary mixing $\left(f_{\mathrm{CBM}}=0\right.$ and with the Ledoux criterion) produces the lowest $\mathrm{CO}$ core mass of $28 \mathrm{M}_{\odot}$. Increasing convective boundary mixing tends to produce higher $\mathrm{CO}$ core masses, however this depends on whether $\mathrm{H}-\mathrm{He}$ shell interactions modify the convective core mass during Helium burning. For instance, $\mathrm{H}-\mathrm{He}$ shell interactions impact the model with $f_{\mathrm{CBM}}=0.01$ at $Z=0.0003$ so that despite the larger overshooting, its final $\mathrm{CO}$ core mass is lower than the model with $f_{\mathrm{CBM}}=0.001$.

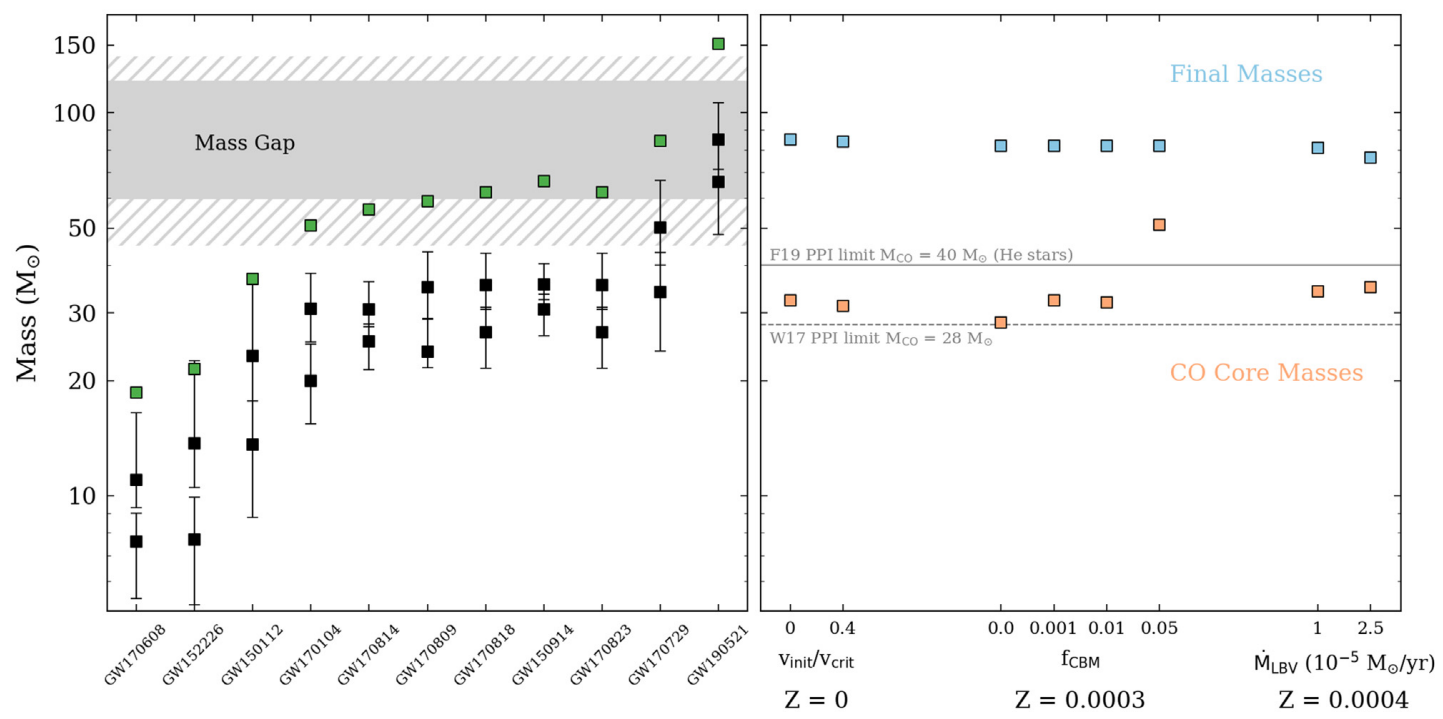

Figure 1. Left-hand panel: Pre-merger and final BH masses from LIGO/Virgo observations in O1/O2 with GW190521 and the predicted region of the mass gap due to pair-instability. Right-hand panel: Final masses (blue) and CO core masses (red) of selected $85 \mathrm{M}_{\odot}$ models listed in Table 1 . We also include the maximum CO core mass found by Woosley (2017) that avoids any pulsations due to pair instability. 


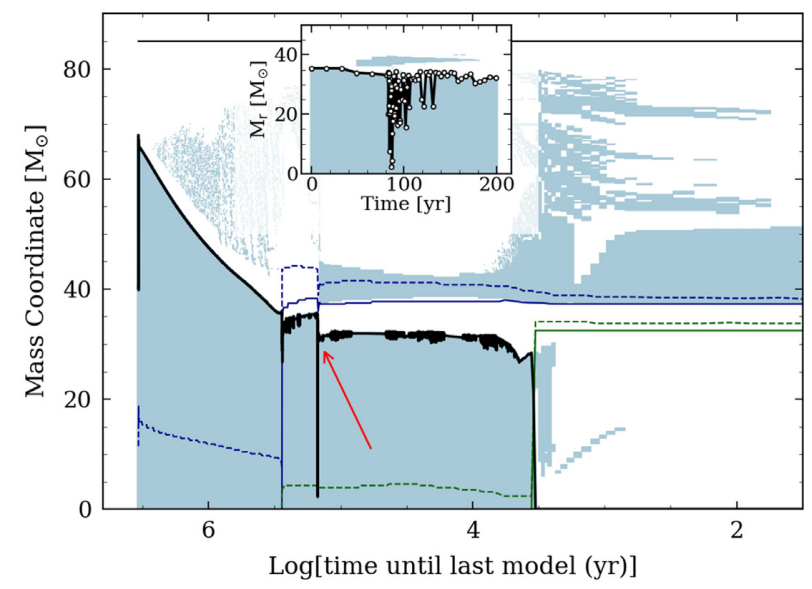

Figure 2. Kippenhahn diagram of a GENEC non-rotating $85 \mathrm{M}_{\odot}$ model at $Z=0$. Solid (dashed) lines correspond to the peak $\left(100 \mathrm{erg} \mathrm{g}^{-1} \mathrm{~s}^{-1}\right)$ of the energy generation rate for $\mathrm{H}$ burning (blue) and He burning (green). The red arrow indicates the $\mathrm{H}-\mathrm{He}$ shell interaction. An inset is included at the top of the figure to show that the interaction is resolved, where white circles indicate each timestep.

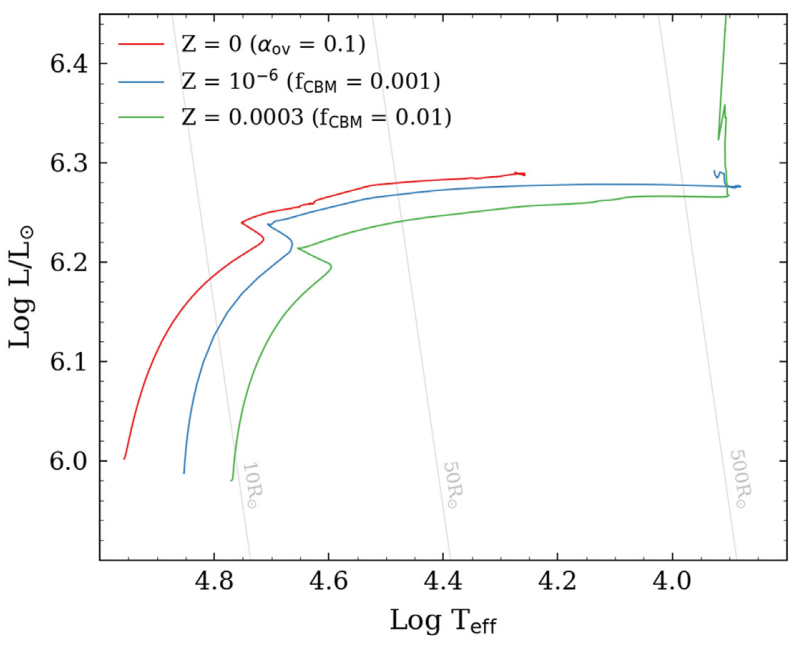

Figure 3. Evolutionary tracks of selected $85 \mathrm{M}_{\odot}$ models in the HertzsprungRussell diagram with $Z=0, Z=10^{-6}$, and $Z=0.0003$.

$\mathrm{H}-\mathrm{He}$ shell interactions are an interesting possibility to reduce the final $\mathrm{CO}$ core masses of massive stars at low and zero $Z$ (Ekström et al. 2008; Clarkson \& Herwig 2020). This is relevant as it may allow a star to avoid the pulsational pair instability regime, depending on initial mass and metallicity. To demonstrate this, we plot the Kippenhahn diagram of the evolution of our non-rotating $85 \mathrm{M}_{\odot} Z=0$ stellar model (Fig. 2). As expected, the convective core mass decreases during the MS evolution and increases following the onset of Heburning. However, shortly after the beginning of He-burning, the $\mathrm{H}$ shell burning region becomes convective. This causes the convective core mass to decrease by $\sim 5 \mathrm{M}_{\odot}$ (inset plot in Fig. 2 ) and prevents any subsequent increase as the star evolves to the end of He-burning.

Fig. 3 shows the evolutionary tracks in the Hertzsprung-Russell diagram of three $85 \mathrm{M}_{\odot}$ models with metallicities of $Z=0,10^{-6}$, and 0.0003 . The qualitative evolution during the MS is similar for all models. The location of the zero-age main-sequence moves to higher $T_{\text {eff }}$ and luminosity with decreasing metallicity due the lower CNO abundances in the core. The post-MS evolution is affected in a similar way by the metallicity. At lower metallicites, a lower $\mathrm{CNO}$ abundance in the hydrogen-burning shell favours a more compact envelope and a higher $T_{\text {eff }}$. This trend continues until the pre-supernova stage, so that the maximum radii that the models reach are 142672 and 794 $\mathrm{R}_{\odot}$ for $Z=0,10^{-6}$, and 0.0003 , respectively.

Previous works have focused on the context of producing BHs in close binary systems that could easily merge in the Hubble time and as a result assume that the entire $\mathrm{H}$ envelope will be lost to some combination of stellar winds, eruptions by luminous blue variables or binary interaction (e.g. Farmer et al. 2019). As a result, they focus on the evolution and deaths of helium stars (e.g. Woosley 2019). For single stars with hydrogen envelopes, a maximum $\mathrm{BH}$ mass of 60-65 $\mathrm{M}_{\odot}$ has been suggested for non-rotating models (Spera \& Mapelli 2017; Woosley 2017; Mapelli et al. 2020). In their models, strong mass-loss of the higher mass models coupled with higher core masses prevented the formation of higher mass BHs. Rotating models were found to have lower maximum BH masses. The models presented in this paper indicate black hole masses of up to 70 $75 \mathrm{M}_{\odot}$, and possibly up to $85 \mathrm{M}_{\odot}$, depending on uncertainties related to convective mixing, mass-loss, $\mathrm{H}-\mathrm{He}$ shell interactions, and pairinstability pulsations. Our models leave open the possibility of a mass gap above $85 \mathrm{M}_{\odot}$. To properly infer the actual limits of the pair instability mass gap based on these models, we would need to compute a large grid of models with different initial masses, rotation rates, and metallicities. We defer this to future work.

\section{IMPLICATIONS FOR BLACK HOLE MASSES FROM THE FIRST STELLAR GENERATIONS}

Our models with $Z=0-0.0004$ have three properties which favour higher BH masses as compared to higher metallicity models. These are (i) lower mass-loss rates, in particular during the post-MS phase, (ii) possible $\mathrm{H}-\mathrm{He}$ shell interactions which lower the $\mathrm{CO}$ core mass, and (iii) a more compact star disfavouring binary interaction.

\subsection{Lower mass-loss during the evolution}

The amount of mass that a star retains until the pre-supernova stage depends strongly on its metallicity (e.g. Groh et al. 2019). This is a result of the strong dependence of mass-loss from radiative-driven winds on metallicity (Vink, de Koter \& Lamers 2001). For solar metallicity stars, the time-averaged mass-loss rate during the LBV phase and the presence of surface magnetic fields are important factors that determine the final $\mathrm{BH}$ mass of massive stars, which can range from 35 to $71 \mathrm{M}_{\odot}$ for an $85 \mathrm{M}_{\odot}$ star (Groh et al. 2020). At low metallicity, mass-loss by stellar winds during the mainsequence phase becomes very low. Our $85 \mathrm{M}_{\odot}$ models at $Z=0.0003$ lose only $1.5 \mathrm{M}_{\odot}$ during the MS assuming the Vink et al. (2001) prescription. Further mass-loss occurs during the post-MS and is strongly dependent on how cool the surface becomes. Our $Z=0.0003$ MESA models stay hot and lose $1.5 \mathrm{M}_{\odot}$ during the post-MS, while our GENEC models can become spectroscopically similar to LBVs (Groh et al. 2014). As a result, they may lose significantly more mass at that stage $\left(7.5 \mathrm{M}_{\odot}\right.$ for $\left.\dot{M}_{\mathrm{LBV}, \max }=2.5 \times 10^{-5} \mathrm{M}_{\odot} \mathrm{yr}^{-1}\right)$, even at low metallicity (Smith \& Owocki 2006; Allan et al. 2020).

At zero metallicity, radiatively driven mass-loss becomes negligible throughout the evolution (Krtička \& Kubát 2006), although for fast-rotating stars, there can be some small mass-loss if the critical rotation limit is reached. Zero or negligible mass-loss has been customarily used in stellar evolution grids at zero metallicity such as Marigo et al. (2001), Ekström et al. (2008), Yoon et al. (2012), Windhorst et al. (2018), and Murphy et al. 2020 (in preparation). As 
such, our zero-metallicity models retain most of their mass until core collapse. There is little observational constraints for mass-loss rates at these extremely low- $Z$ values, in particular for the post-MS stages, and we should regard our assumptions about mass-loss rates as highly uncertain. Uncertainties related to mass-loss rates may affect both the final mass, the $\mathrm{CO}$ core mass, and the maximum radius.

\subsection{Possibility of $\mathrm{H}-\mathrm{He}$ shell interactions}

Some of our models at low/zero metallicity experience strong $\mathrm{H}-$ He shell interactions (Fig. 2). This behaviour has been seen in previous low metallicity stellar evolution models (e.g. Chieffi \& Limongi 2004; Ekström et al. 2008; Ritter et al. 2018; Clarkson \& Herwig 2020). During He-burning, a low or zero abundance of CNO elements in the H-burning shell favours a bluer star which increases the likelihood of the $\mathrm{H}$-burning region becoming convective and subsequently reducing the convective core mass. In models with $Z=0$, diffusion of $\mathrm{C}$ from the He-burning core to the $\mathrm{H}$ burning shell can trigger a strong $\mathrm{CNO}$ cycle boost, make the shell convective and lead to $\mathrm{H}-\mathrm{He}$ shell interactions. By comparing the GENEC models for metallicites of $Z=0.0004,0.002$, and 0.014 , Groh et al. (2019) discuss that the occurrence of $\mathrm{H}-\mathrm{He}$ shell interactions may be favoured at lower metallicities. Clarkson \& Herwig (2020) find different types of $\mathrm{H}-\mathrm{He}$ shell interactions that occur at different times during the evolution. Some of these interactions, particularly during the late stages, may dramatically reduce the $\mathrm{CO}$ core mass and allow the star to avoid the pulsational pair instability regime. We encourage further work on the effects of convective boundary mixing and rotation on $\mathrm{H}-\mathrm{He}$ shell interactions as this is crucial for understanding the fate of massive stars at low and zero metallicity.

Some of our models assume a relatively low amount of convective overshooting. The extent and implementation of convective overshooting in stellar models has a large impact on the mass of the $\mathrm{He}$ and CO cores (e.g. Kaiser et al. 2020). Three-dimensional models of lower mass stars favour the existence of such mixing at convective boundaries (e.g. Cristini et al. 2017), although it is still unclear how it is affected by other parameters such as mass and metallicity. In addition, for stars of initial mass $7 \mathrm{M}_{\odot}<M_{\text {init }}<25 \mathrm{M}_{\odot}$ a high value of $f_{\mathrm{CBM}}$ is favoured (Martinet et al. 2020, in preparation) as well as for masses of $\sim 35 \mathrm{M}_{\odot}$ (Higgins \& Vink 2019). However, these constraints are for core- $\mathrm{H}$ burning stars. The value of $f_{\mathrm{CBM}}$ is not as well constrained for other burning phases or for stars of $\sim 85 \mathrm{M}_{\odot}$ which have different internal structures to $\sim 15 \mathrm{M}_{\odot}$ stars and larger core mass ratios.

\subsection{Smaller radius disfavours binary interaction}

Zero-metallicity models favour the retention of the H-envelope in binary systems because they are more compact than higher metallicity stars. For example, the maximum radius of our $85 \mathrm{M}_{\odot}$ rotating model at $\mathrm{Z}=0$ is $R_{\max }=142 \mathrm{R}_{\odot}$, as compared to $952 \mathrm{R}_{\odot}$ at $\mathrm{Z}=0.0004$, and $815 \mathrm{R}_{\odot}$ at $\mathrm{Z}=0.014$. The radius of stellar models at these masses depends greatly on the assumptions for convection in the envelope (e.g. Gräfener, Owocki \& Vink 2012; Jiang et al. 2018). Additionally, the radius is strongly impacted by uncertainties related to the chemical abundance profile in the envelope (Farrell et al. 2020), which is impacted by the properties of mixing (e.g. Schootemeijer et al. 2019). The size and interaction of convective shells above the core during the MS and between the MS and He-burning greatly affect the radius of the star during He-burning. If these processes result in hydrogen being mixed into the $\mathrm{H}$-shell burning region, the star will remain more compact for longer during He-burning.
Binary interactions may also provide a mechanism to produce a pre-supernova structure with a high hydrogen envelope mass (e.g. Justham, Podsiadlowski \& Vink 2014). Mass gainers or products of mergers during the post-MS that do not fully rejuvenate could have low core masses and large envelope masses, potentially avoiding the PPI regime and collapsing to a black hole with the $\mathrm{H}$ envelope falling back on to the BH (Di Carlo et al. 2019; Spera et al. 2019).

\subsection{Pulsational pair-instability}

Models suggest that stars with a CO core mass of $\gtrsim 28 \mathrm{M}_{\odot}$ will undergo pair-instability driven pulsation during their final stages (Woosley 2017). For example, Woosley (2017) present a model (T80D) with a final mass of $80 \mathrm{M}_{\odot}$ and a CO core mass of $32.6 \mathrm{M}_{\odot}$ that, due to pulsations, will produce a final $\mathrm{BH}$ mass of $34.9 \mathrm{M}_{\odot}$. The exact value of the maximum $\mathrm{CO}$ core mass of this boundary that will avoid the pair-instability is uncertain (e.g. Woosley 2017; Farmer et al. 2019; Marchant et al. 2019) and effects related to convective boundary mixing, stellar winds, and the ${ }^{12} \mathrm{C}(\alpha, \gamma){ }^{16} \mathrm{O}$ reaction rate may increase this value. Our $60 \mathrm{M}_{\odot}$ models with $\mathrm{Z}=0$ have $\mathrm{CO}$ core masses between 21 and $24 \mathrm{M}_{\odot}$. Most of our $85 \mathrm{M}_{\odot}$ models are just above this strict limit with $\mathrm{CO}$ core masses of 31-35 $\mathrm{M}_{\odot}$. We computed a test model with no convective boundary mixing that finishes with a $\mathrm{CO}$ core mass of $28 \mathrm{M}_{\odot}$. By interpolating between our 60 and $85 \mathrm{M}_{\odot}$ models, we compute that a $72 \mathrm{M}_{\odot}$ model will have a final $\mathrm{CO}$ core mass of $28 \mathrm{M}_{\odot}$ under the standard assumptions for convection in the GENEC models.

For a pulse of a given energy, the amount of mass that a star loses depends on the binding energy of the envelope. More compact, hotter stars are less likely to lose their entire $\mathrm{H}$ envelope compared to extended envelopes, such as in red supergiants. For this reason, $Z=0$ models are favoured to retain large masses as they remain compact until the end of their evolution. Farmer et al. (2019) find a CO core mass limit for the onset of PPI of $\sim 40 \mathrm{M}_{\odot}$ for highly compact helium stars. Since our models are hydrogen rich, with a lower binding energy than helium stars, it is unclear if this limit would apply to our $85 \mathrm{M}_{\odot}$ models. Further studies could investigate the impact of the uncertainties discussed by Farmer et al. (2019), such as the ${ }^{12} C(\alpha, \gamma){ }^{16} O$ reaction rate, in hydrogen-rich models that are blue and relatively compact, such as our $Z=0$ models. If the pulses are not present and/or do not remove the $\mathrm{H}$ envelope, this may allow the formation of $85 \mathrm{M}_{\odot} \mathrm{BHs}$.

\section{IMPACTS FOR BINARY BLACK HOLE MERGERS}

Due to their lower mass-loss rates, smaller radii and the possibility of $\mathrm{H}-\mathrm{He}$ shell interactions that reduce the $\mathrm{CO}$ core mass, stars in the first stellar generations are ideal candidates to produce $\mathrm{BHs}$ in the mass gap such as GW190521, with masses of 70-75 $\mathrm{M}_{\odot}$. In order to produce a $\mathrm{BBH}$ merger observable by LIGO/Virgo, such a $\mathrm{BH}$ would need to be in a close binary system. Due to uncertainties in the evolution of massive stars and in how these stars behave in binary systems, it is difficult to perfectly constrain the possible evolutionary pathways that would lead to a system. Despite their large H-envelope mass, our models at $Z=0$ expand only to radii $\sim 100 \mathrm{R}_{\odot}$. If the star has a binary companion and avoids Roche-Lobe overflow, the merging timescale would likely exceed the Hubble time. However, if the orbital separation were to reduce after the more massive star dies (e.g. due to a common envelope phase), this may reduce the merging timescale. Alternatively, if the BH is in a dense stellar cluster, it could dynamically capture a companion and form a close binary system 
(e.g. Sigurdsson \& Hernquist 1993; Portegies Zwart \& McMillan 2000; Downing et al. 2010; Rodriguez et al. 2016). We leave the details of the binary evolution scenario or dynamical capture to future work (e.g. Belczynski 2020).

\section{ACKNOWLEDGEMENTS}

EF, JHG, and LM thank the Irish Research Council for funding. JHG thanks Isidoros and Giorgos for providing useful resources. This article is based upon work from the 'ChETEC' COST Action (CA16117). RH acknowledges support from the IReNA AccelNet Network of Networks (NSF grant no. OISE-1927130) and from the World Premier International Research Centre Initiative. CG has received funding from the ERC (grant no. 833925).

\section{DATA AVAILABILITY}

The derived data generated in this research will be shared on reasonable request to the corresponding author.

\section{REFERENCES}

Abbott B. P. et al., 2019, Phys. Rev. X, 9, 031040

Allan A. P., Groh J. H., Mehner A., Smith N., Boian I., Farrell E. J., Andrews J. E., 2020, MNRAS, 496, 1902

Belczynski K. et al., 2016, A\&A, 594, A97

Belczynski K., 2020, ApJL, 905, L15

Belczynski K. et al., 2020, ApJ, 890, 113

Chatzopoulos E., Wheeler J. C., 2012, ApJ, 748, 42

Chen K.-J., Woosley S., Heger A., Almgren A., Whalen D. J., 2014, ApJ, 792, 28

Chieffi A., Limongi M., 2004, ApJ, 608, 405

Choi J., Dotter A., Conroy C., Cantiello M., Paxton B., Johnson B. D., 2016, ApJ, 823, 102

Clarkson O., Herwig F., 2020, preprint (arXiv:2005.07748)

Cristini A., Meakin C., Hirschi R., Arnett D., Georgy C., Viallet M., Walkington I., 2017, MNRAS, 471, 279

Croon D., McDermott S. D., Sakstein J., 2020, preprint (arXiv:2007.07889)

De Luca V., Desjacques V., Franciolini G., Pani P., Riotto A., 2020, preprint (arXiv:2009.01728)

Di Carlo U. N., Giacobbo N., Mapelli M., Pasquato M., Spera M., Wang L., Haardt F., 2019, MNRAS, 487, 2947

Downing J. M. B., Benacquista M. J., Giersz M., Spurzem R., 2010, MNRAS, 407, 1946

Ekström S., Meynet G., Chiappini C., Hirschi R., Maeder A., 2008, A\&A, 489,685

Ekström S. et al., 2012, A\&A, 537, A146

Farmer R., Renzo M., de Mink S. E., Marchant P., Justham S., 2019, ApJ, 887,53

Farmer R., Renzo M., de Mink S., Fishbach M., Justham S., 2020, ApJ, 902 , 36

Farr W. M., Fishbach M., Ye J., Holz D. E., 2019, ApJ, 883, L42

Farrell E. J., Groh J. H., Meynet G., Eldridge J. J., Ekström S., Georgy C., 2020, MNRAS, 495, 4659

Fishbach M., Holz D., 2020, ApJL, 904, 26

Fishbach M., Holz D. E., Farr B., 2017, ApJ, 840, L24

Fowler W. A., Hoyle F., 1964, ApJS, 9, 201

Fragione G., Loeb A., Rasio F., 2020, ApJ, 902, L26

Fryer C. L., Woosley S. E., Heger A., 2001, ApJ, 550, 372

Gayathri V. et al., 2020, preprint (arXiv:2009.05461)

Georgy C. et al., 2013, A\&A, 558, A103

Gerosa D., Berti E., 2017, Phys. Rev. D, 95, 124046

Giacobbo N., Mapelli M., Spera M., 2018, MNRAS, 474, 2959

Glatzel W., Fricke K. J., El Eid M. F., 1985, A\&A, 149, 413

Gräfener G., Owocki S. P., Vink J. S., 2012, A\&A, 538, A40
Groh J. H. et al., 2019, A\&A, 627, A24

Groh J. H., Meynet G., Ekström S., Georgy C., 2014, A\&A, 564, A30

Groh J. H., Farrell E. J., Meynet G., Smith N., Murphy L., Allan A. P., Georgy C., Ekstroem S., 2020, ApJ, 900, 98

Heger A., Fryer C. L., Woosley S. E., Langer N., Hartmann D. H., 2003, ApJ, 591, 288

Higgins E. R., Vink J. S., 2019, A\&A, 622, A50

Jiang Y.-F., Cantiello M., Bildsten L., Quataert E., Blaes O., Stone J., 2018, Nature, 561, 498

Justham S., Podsiadlowski P., Vink J. S., 2014, ApJ, 796, 121

Kaiser E. A., Hirschi R., Arnett W. D., Georgy C., Scott L. J. A., Cristini A., 2020, MNRAS, 496, 1967

Kasen D., Woosley S. E., Heger A., 2011, ApJ, 734, 102

Kinugawa T., Nakamura T., Nakano H., 2020a, preprint (arXiv:2009.06922)

Kinugawa T., Nakamura T., Nakano H., 2020b, MNRAS, 498, 3946

Krtička J., Kubát J., 2006, A\&A, 446, 1039

Leung S.-C., Nomoto K., Blinnikov S., 2019, ApJ, 887, 72

Mapelli M., Spera M., Montanari E., Limongi M., Chieffi A., Giacobbo N., Bressan A., Bouffanais Y., 2020, ApJ, 888, 76

Marchant P., Moriya T. J., 2020, A\&A, 640, L18

Marchant P., Renzo M., Farmer R., Pappas K. M. W., Taam R. E., de Mink S. E., Kalogera V., 2019, ApJ, 882, 36

Marigo P., Girardi L., Chiosi C., Wood P. R., 2001, A\&A, 371, 152

Miller M. C., Hamilton D. P., 2002, MNRAS, 330, 232

Ober W. W., El Eid M. F., Fricke K. J., 1983, A\&A, 119, 61

Paxton B. et al., 2013, ApJS, 208, 4

Paxton B. et al., 2015, ApJS, 220, 15

Paxton B., Bildsten L., Dotter A., Herwig F., Lesaffre P., Timmes F., 2011, ApJS, 192, 3

Portegies Zwart S. F., McMillan S. L. W., 2000, ApJ, 528, L17

Rakavy G., Shaviv G., Zinamon Z., 1967, ApJ, 150, 131

Renzo M., Farmer R. J., Justham S., de Mink S. E., Götberg Y., Marchant P., 2020, MNRAS, 493, 4333

Ritter C., Herwig F., Jones S., Pignatari M., Fryer C., Hirschi R., 2018, MNRAS, 480, 538

Rodriguez C. L., Zevin M., Pankow C., Kalogera V., Rasio F. A., 2016, ApJ, 832, L2

Rodriguez C. L., Zevin M., Amaro-Seoane P., Chatterjee S., Kremer K., Rasio F. A., Ye C. S., 2019, Phys. Rev. D, 100, 043027

Romero-Shaw I. M., Lasky P. D., Thrane E., Calderon Bustillo J., 2020, preprint (arXiv:2009.04771)

Sakstein J., Croon D., McDermott S. D., Straight M. C., Baxter E. J., 2020, preprint (arXiv:2009.01213)

Schootemeijer A., Langer N., Grin N. J., Wang C., 2019, A\&A, 625, A132

Sigurdsson S., Hernquist L., 1993, Nature, 364, 423

Smith N., Owocki S. P., 2006, ApJ, 645, L45

Spera M., Mapelli M., 2017, MNRAS, 470, 4739

Spera M., Mapelli M., Giacobbo N., Trani A. A., Bressan A., Costa G., 2019, MNRAS, 485, 889

Stevenson S., Sampson M., Powell J., Vigna-Gómez A., Neijssel C. J., Szécsi D., Mandel I., 2019, ApJ, 882, 121

Tanikawa A., Susa H., Yoshida T., Trani A. A., Kinugawa T., 2020, preprint (arXiv:2008.01890)

The LIGO Scientific Collaboration, 2020a, preprint (arXiv:2009.01075)

The LIGO Scientific Collaboration, 2020b, preprint (arXiv:2009.01190)

Umeda H., Nomoto K., 2002, ApJ, 565, 385

van Son L. A. C. et al., 2020, ApJ, 897, 100

Vink J. S., de Koter A., Lamers H. J. G. L. M., 2001, A\&A, 369, 574

Windhorst R. A. et al., 2018, ApJS, 234, 41

Woosley S. E., 2017, ApJ, 836, 244

Woosley S. E., 2019, ApJ, 878, 49

Woosley S. E., Blinnikov S., Heger A., 2007, Nature, 450, 390

Yoon S. C., Dierks A., Langer N., 2012, A\&A, 542, A113

This paper has been typeset from a $\mathrm{T}_{\mathrm{E}} \mathrm{X} / \mathrm{LT} \mathrm{E} \mathrm{X}$ file prepared by the author. 